\title{
CONTROLE DE Myzus persicae (SULZER 1778) (HOMOPTERA, APHIDIDAE) EM BATATA (Solanum tuberosum) ( L.)
}

\author{
Control of Myzus persicae (Sulzer 1778) (Homoptera: Aphididae) \\ in potato (Solanum tuberosum) (L.)
}

\footnotetext{
Rui Scaramella Furiatti ${ }^{1}$, Airton Rodrigues Pinto Jr. ${ }^{2}$, Paulo Roberto Valle Silva Pereira ${ }^{3}$

${ }^{1}$ Engenheiro Agrônomo, Dr., Professor Adjunto do Departamento de Agronomia, Universidade Estadual de Ponta Grossa. Ponta Grossa, PR - Brasil.e-mail: furiatti@convoy.com.br

2 Engenheiro Agrônomo, Dr., Professor Adjunto III do Curso de Agronomia. Pontifícia Universidade Católica do Paraná PUCPR.Curitiba, PR - Brasil.e-mail: airton.junior@pucpr.br.

${ }^{3}$ Engenheiro Agrônomo, Dr., Pesquisador da Embrapa RR - Laboratório de Entomologia. Boa Vista, RR - Brasil. e-mail: paulo@cpafrr.embrapa.br
}

\section{Resumo}

Foi testada a eficiência de inseticidas granulados no controle de Myzus persicae em uma cultura de batata no estado do Paraná, Sul do Brasil. Os tratamentos foram dispostos em 4 blocos com 8 parcelas cada, com espaçamento de 3,2 × 3,5 m e distribuídos ao acaso. Nos tratamentos com a dosagem parcelada, metade foi aplicada durante o plantio e o restante 20 dias após a emergência das plantas. As avaliações foram realizadas 19, 27 e 35 dias após a emergência, usando a metodologia das 3 folhas. Fosthiazate ( 20 e $30 \mathrm{~kg}^{-\mathrm{ha}^{-1}}$ ), phorate $\left(50 \mathrm{~kg} \cdot \mathrm{ha}^{-1}\right)$ e aldicarb $\left(13\right.$ e $\left.6,5+6,5 \mathrm{~kg} \cdot \mathrm{ha}^{-1}\right)$ foram efetivos até os 27 dias após a emergência; fosthiazate (40 e $\left.20+20 \mathrm{~kg} \cdot h \mathrm{ha}^{-1}\right)$ e aldicarb $\left(13 \mathrm{~kg} \cdot \mathrm{ha}^{-1}\right)$ foram eficazes no controle de $M$. persicae até 33 dias após a emergência.

Palavras-chave: Pulgão verde da batata; Controle químico; Inseticidas de solo.

\begin{abstract}
The efficiency of granulated insecticides was tested against Myzus persicae in a potato crop in Parana State, Southern Brazil. The treatments were arranged in 4 blocks with 8 plots each, with $3.2 \times 3.5 \mathrm{~m}$, randomly selected. In the treatments with parceled dosage, one half was applied during planting, and the other 20 days after the plant emergence. The evaluations were made 19,27 and 33 days after emergence, using the three leaves method. Fosthiazate (20 and $30 \mathrm{~kg} / \mathrm{ha})$, phorate $(50 \mathrm{~kg} / \mathrm{ha}$ ) and aldicarb (13 and $6.5+6.5 \mathrm{~kg} / \mathrm{ha}$ ) were effective up to 27 days after emergence; fosthiazate (40 and $20+20 \mathrm{~kg} / \mathrm{ha})$ and aldicarb $(13 \mathrm{~kg} / \mathrm{ha}$ ) were effective against M. persicae up to 33 days after emergence.
\end{abstract}

Keywords: Green peach aphid; Chemical control; Soil insecticide. 


\section{INTRODUÇÃO}

A batata, Solanum tuberosum (Lineu), é uma das principais fontes de alimento da humanidade, sendo suplantada apenas pelo arroz e pelo trigo, em que o milho é um cereal mais utilizado em nutrição animal. Trata-se de uma cultura cosmopolita. No Brasil, ocupa o primeiro lugar em importância econômica entre as culturas oleráceas.

Esta cultura apresenta muitos problemas fitossanitários, dentre os quais, os insetos, cujos danos podem acarretar perdas significativas, afetando o rendimento e também a qualidade.

O controle das pragas da batata assume dimensões distintas, dependendo da finalidade do cultivo. Para batata consumo podem ser tolerados níveis populacionais mais altos de certas pragas do que para produção de batata semente. No caso da batata semente, o nível populacional de afídeos é de extrema importância, visto que esses insetos são conhecidos transmissores de viroses.

Myzus persicae (SULZER, 1778), pulgão verde da batata (PVB), é capaz de transmitir mais de 100 viroses de plantas em aproximadamente 30 famílias, incluindo culturas de caráter econômico no mundo inteiro (VAN EMDEN et al., 1968). Esse é considerado como o mais importante vetor do PLRV (Potato Leaf Roll Virus) e do PVY (Potato Virus Y), (O' LOUGHLIN, 1962, RADCLIFFE, 1982; VAN HARTEN, 1983). Sekhon, Bindra e Chahal. (1983) demonstraram que aldicarb e forato foram altamente efetivos contra PVB em rabanete. Rice, Stribley e Sawicki (1984) observaram a redução na sobrevivência de PVB e também da sua habilidade em transmitir vírus em couve chinesa com o uso de aldicarb. Esses autores sugeriram que houve um efeito de repelência sobre esse afídeo.

O nível de mortalidade de PVB alados, em plantas de batata tratadas com aldicarb em várias dosagens, requereu um mínimo de 30 horas para atingir 50\% (BOITEAU; KING; LEVESQUE, 1985). A aplicação de aldicarb no plantio e na pós-emergência foi experimentada por vários autores. Labh, Rataul e Singh (1990) utilizando batata, observaram que aldicarb, aplicado no plantio, foi altamente efetivo contra o PVB, também no parcelamento, utilizando-se metade da dosagem no plantio e metade após 35 dias da emergência, o efeito inseticida manteve-se, e houve redução de infecção virótica. Vernon e Outman (1983) encontraram resultados semelhantes ao constatar que aldicarb foi efetivo contra o PVB, por quatro meses em aspargo, quando aplicado em pós-emergência.

Resultados discordantes foram apresentados por Woodford et al. (1984), os quais demonstraram que baixas ou altas dosagens deste inseticida, aplicadas no sulco de plantio, foram superiores à baixa dosagem utilizada em cobertura na cultura da batata. Igualmente Woodford et al. (1988) afirmaram que a técnica de aplicação em pós-emergência de aldicarb é pouco confiável e não recomendada para substituir a aplicação da dosagem total no sulco de plantio. A eficiência do forato contra o PVB foi atestada por Sekhon, Bindra e Chahal (1983), em rabanete e por Toba e Powell (1986); Misra e Agrawal (1988); Trivedi e Misra (1989); Kathpal et al. (1983) em batata, onde observaram que o número de PVB permaneceu abaixo de 20 indivíduos/100 folhas, nível considerado por eles como abaixo do nível de dano econômico. Porém Misra e Agrawal (1990) constataram que o forato não controlou o PVB, durante todo o ciclo da cultura da batata, quando aplicado no plantio e sem o uso de inseticidas na parte aérea.

Neste trabalho, avaliou-se a atividade biológica de inseticidas de solo sobre M. persicae, durante o estágio de crescimento de plantas de batata. 


\section{MATERIAL E MÉTODOS}

O experimento foi realizado na Fazenda Experimental da Nutrimental S.A., no município de São José dos Pinhais, Paraná. O delineamento estatístico utilizado foi em blocos casualizados, composto de quatro blocos de oito parcelas de $11,2 \mathrm{~m}^{2}$ cada, distribuídas ao acaso. O plantio foi realizado no dia 19 de outubro de 2003, com tubérculos tipo 2 da cultivar Atlantic, espaçados de 0,8 $\mathrm{m}$ entre linhas por 0,35 m entre plantas. Utilizou-se $3 \mathrm{t}$ de adubo $4-14-8$ por ha.

Os tratamentos foram feitos manualmente no sulco de plantio, utilizando-se o produto pesado para 3,5 m lineares, acondicionado em sacos plásticos, correspondentes ao comprimento da linha de cada parcela. Nos tratamentos foram utilizados os carbamatos, aldicarb (Temik ${ }^{\circledR} 150$ nas dosagens de 13 e 6,5 + 6,5 kg de produto comercial (p.c.).ha ${ }^{-1}$ ), fosthiazate (IKI $114510 \%$ nas dosagens de 20, 30, 40 e $20+$ $20 \mathrm{~kg}$ p.c. ha ${ }^{-1}$ ) e o organofosforado forato (Granutox ${ }^{\mathrm{R}}$, na dosagem de $50 \mathrm{~kg}$ p.c. ha ${ }^{-1}$ ). A segunda aplicação dos produtos que tiveram sua dosagem parcelada (fosthiazate $20+20 \mathrm{~kg}$. ha-1 e aldicarb $6,5+6,5 \mathrm{~kg}$ p.c. $\mathrm{ha}^{-1}$ ) foi realizada 20 dias após a emergência das plantas, por ocasião da amontoa.

Os tratamentos foram avaliados conforme Furiatti e Almeida (1992), pelo número de pulgões vivos por planta com auxílio do método das três folhas, exceto na primeira avaliação, que por se tratar de plantas menores que $20 \mathrm{~cm}$ de altura, contou-se o número total de insetos por planta. A contagem de afídeos foi realizada em quatro plantas por parcela aos 19, 27 e 33 dias após a emergência.

Os dados obtidos foram submetidos à análise de variância e as diferenças entre as médias tratadas pelo teste de Duncan 5\%. A eficiência relativa dos inseticidas foi calculada segundo Abbott (1925).

A eficiência no controle de Myzus persicae foi atribuída aos inseticidas que apresentaram mortalidade superior a $80 \%$ e diferença estatística da testemunha.

\section{RESULTADOS E DISCUSSÃO}

Verificou-se que nas duas primeiras avaliações, aos 19 e 27 dias após a emergência, não diferiram estatisticamente entre si fosthiazate (nas dosagens de 30, $40 \mathrm{~kg}$. ha ${ }^{-1}$ e $20+20 \mathrm{~kg}$ p.c. ha ${ }^{-1}$ ), forato e aldicarb (nas dosagens testadas). Porém, após 33 dias da emergência, apenas fosthiazate (dosagens de 40 e $20+20 \mathrm{~kg}$ p.c. ha ${ }^{-1}$ ) e aldicarb (na dosagem de $13 \mathrm{~kg}$ p.c. ha ${ }^{-1}$ ) foram eficazes contra o PVB. Também se observou que fosthiazate (dosagem de $20 \mathrm{~kg}$ p.c. ha ${ }^{-1}$ ) e o forato não mostraram diferença significativa da testemunha (TABELA 1). A eficiência de aldicarb foi constatada por Sekhon, Bindra e Chahal (1983), Vernon e Houtman (1983); Rice, Stribley e Sawicki (1984); Woodford et al. (1984) e Labh, Rataul e Singh (1990). Aldicarb utilizado parceladamente no plantio e em cobertura, perdeu a eficácia após 33 dias da emergência, condição também observada em trabalhos de Woodford et al. $(1984,1988)$ os quais afirmaram que a técnica de aplicação de aldicarb em pós-emergência é pouco confiável e não-recomendável. Porém, discordando dos resultados observados no trabalho de Labh, Rataul e Singh (1990), os quais obtiveram bons resultados no controle do PVB e de doenças viróticas, com o parcelamento deste inseticida. Fosthiazate, na dosagem parcelada, $\left(20+20 \mathrm{~kg}\right.$ p.c. ha $\left.{ }^{-1}\right)$, mostrou resultado semelhante ao obtido com aldicarb, tendo a sua eficácia reduzida de $100 \%$ para $83,8 \%$, do $27^{\circ}$ dia para o $33^{\circ}$ dia após a emergência das plantas. Os resultados obtidos com forato foram semelhantes aos encontrados por Kathpal et al. (1983); Toba e Powell (1986); Misra e Agrawal (1988); Triverdi e Misra (1989). 
TABELA 1 - Número médio de Myzus persicae por planta (X) e porcentagem de eficiência de inseticida (E\%) em diferentes avaliações após a emergência

Table 1 - Average number of Myzus persicae in plants $(x)$ and percentage of insecticide efficiency (E\%) in different evaluations after plant emergency

\begin{tabular}{|c|c|c|c|c|c|c|c|c|}
\hline \multirow[t]{3}{*}{ Tratamento } & \multirow[t]{3}{*}{$\begin{array}{l}\text { Nome } \\
\text { Comercial }\end{array}$} & \multirow{3}{*}{$\begin{array}{l}\text { Dose } \\
(\mathrm{Kg} \mathrm{de} \\
\left.\text { p.c..ha }{ }^{-1}\right)\end{array}$} & & & \multicolumn{4}{|c|}{ Dias após a emergência } \\
\hline & & & \multicolumn{2}{|c|}{19 dias } & \multicolumn{2}{|c|}{27 dias } & \multicolumn{2}{|c|}{33 dias } \\
\hline & & & $\mathrm{x}^{1 /}$ & $\mathrm{E} \%$ & $\mathrm{X}$ & $\mathrm{E} \%$ & $\mathrm{X}$ & $\mathrm{E} \%$ \\
\hline 1 & IKI $1145^{2 /}$ & 20,0 & $1,18 \mathrm{bc}$ & 62,9 & $1,68 \mathrm{~cd}$ & 85,2 & $9,87 \mathrm{abc}$ & 22,6 \\
\hline 2 & IKI 1145 & 30,0 & $0,25 \mathrm{~cd}$ & 92,1 & $0,75 \mathrm{~cd}$ & 93,4 & $3,25 \mathrm{bcd}$ & 74,5 \\
\hline 3 & IKI 1145 & 40,0 & $0,12 \mathrm{~d}$ & 96,2 & $0 \mathrm{~d}$ & 100,0 & $0,25 \mathrm{~d}$ & 98,0 \\
\hline 4 & IKI 1145 & $20,0+20,0$ & $0,18 \mathrm{~cd}$ & 94,3 & $0 \mathrm{~d}$ & 100,0 & $2,06 \mathrm{~cd}$ & 83,8 \\
\hline 5 & Granutox ${ }^{\circledR}$ & 50,0 & $0,06 \mathrm{~d}$ & 98,1 & $0,90 \mathrm{~cd}$ & 92,0 & $8,25 \mathrm{abc}$ & 35,3 \\
\hline 6 & Temik $® 150$ & 13,0 & $0,06 \mathrm{~d}$ & 98,1 & $0,75 \mathrm{~cd}$ & 93,0 & $1,0 \mathrm{~d}$ & 92,1 \\
\hline 7 & Temik $® 150$ & $6,5+6,5$ & $0 \mathrm{~d}$ & 100,0 & $0,18 \mathrm{~d}$ & 98,4 & $2,81 \mathrm{~cd}$ & 77,9 \\
\hline 8 & Testemunha & $-\quad 3,18 \mathrm{a}$ & - & $11,37 \mathrm{a}$ & - & $12,75 \mathrm{ab}$ & - & \\
\hline CV \% & & & \multicolumn{2}{|c|}{86,00} & \multicolumn{2}{|c|}{69,86} & \multicolumn{2}{|c|}{53,80} \\
\hline $\mathrm{F}$ & & & \multicolumn{2}{|c|}{5,3840} & \multicolumn{2}{|c|}{7,9798} & \multicolumn{2}{|c|}{4,6434} \\
\hline
\end{tabular}

\section{CONCLUSÕES}

É possível afirmar que apenas fosthiazate aplicado no plantio $\left(40 \mathrm{~kg}\right.$ p.c. ha $\left.{ }^{-1}\right)$ e parcelado $\left(20+20 \mathrm{~kg}\right.$ p.c. ha $\left.{ }^{-1}\right)$ e aldicarb aplicado no plantio $\left(13 \mathrm{~kg}\right.$ p.c. ha $\left.{ }^{-1}\right)$ foram eficientes contra o PVB até os 33 dias após a emergência das plantas.

\section{REFERÊNCIAS}

ABBOT'T, W. S. A method of computing the effectiveness of an insecticide. Journal of Economic Entomology, River Side, CA, v. 18, p. 265-267, 1925.

ANSCOMBE, F. J. On estimating the population of aphids in a potato field. Annals of Applied Biology, Wellesbourne, UK, v. 35, p. 567-571, 1948.

BOITEAU, G.; KING, R. R.; LEVESQUE, D. Lethal and sublethal effects of aldicarb on two potato aphids (Homoptera: Aphididae): Myzus persicae (Sulzer) and Macrosiphum euphorbiae (Thomas). Journal of Economic Entomology, River Side, CA, v. 78, p. 41-44, 1985.

KATHPAL, T. S. et al. Persistence of phorate in soil and its translocation into potato seed tubers. Pesticides, United States, v. 17, p. 39-43, 1983.

LABH, S.; RATAUL, H. S.; SINGH, L. Influence of soil systemic granular insecticides on the mortality of Myzus persicae (Sulzer) and uptake of potato leaf roll virus. Indian Journal of Entomology, New Delhi, v. 52, p. 557-564, 1990.

MISRA, S. S.; AGRAWALL H. O. Chemical control of green peach aphid Myzus persicae (Sulzer) on potato. Journal of Aphidology, India, v. 2, p. 40-50, 1988. 
MISRA, S. S.; AGRAWALL, H. O. Chemical control of green aphid, Myzus persicae (Sulzer) on potato in north-eastern plains. Indian Journal Plant Protection, India, v. 18, p. 11-16, 1990.

O'LOUGHLIN, G. T. Aphid trapping in Victoria. I-The seasonal occurrence of aphids in three localities and a comparison of two methods. Australian Journal of Agriculture Research, Collingwood, v. 14, p. 61-69, 1962.

RADCLIFFE, E. B. Insect pests of potato. Annual Review of Entomology, Palo Alto, v. 27, p. 173-204, 1982.

RICE, A. D.; STRIBLEY, M. F.; SAWICKI, R. M. Efficacy of aldicarb against susceptible and resistant Myzus persicae. In: BRITISH CROP PROTECTION CONFERENCE, 1984, Proceedings... [S:1: s.n.]. 1984. p. 222-228.

SEKHON, S. S.; BINDRA, O. S.; CHAHAL, B. S. Studies on the control of aphids infesting seed crop of radish with soil granular systemic insecticides. Indian Journal Plant Protection, India, v. 11, p. 9-12, 1983.

TOBA, H. H.; POWELL, D. M. Soil aplication of insecticides for controlling three insect pests of potatoes in Washington. Journal of Agricultural Entomology, South Carolina, v. 3, p. 87-99, 1986.

TRIVEDI, T. P.; MISRA, S. S. Chemical control of Myzus persicae (Sulzer) on potato in Deccan Plateau of Maharashtra. Indian Journal Plant Protection, India, v. 17, p. 109-111, 1989.

VAN EMDEN, H. F. et al. The ecology of Myzus persicae. Annual Review of Entomology, Palo Alto, v. 13, p. 197-243, 1968.

VAN HARTEN, A. The relation between aphid fligths and the spread of potato $\mathrm{y}^{\mathrm{N}}\left(\mathrm{PVY}^{\mathrm{N}}\right)$ in the Netherlands. Potato Research, Wageningen, v. 26, p. 01-05, 1983.

VERNON, R. S.; HOUTMAN, R. Evaluation of sprayed and granular aphicides against the European asparagus aphid, Brachycolus asparagi (Homoptera: Aphididae) in British Columbia. Journal of Entomology Society British Columbia, Kamloops, v. 80, p. 3-9, 1983.

WOODFORD, J. A. T. et al. Trials with granular insecticides for the control of aphids and potato leaf roll virus disease in Scotland, 1980-82. Crop protection in northern Britain, Invergowrie, p. 171-176, 1984.

WOODFORD, J. A. T.; GORDON, S. C.; FOSTER, G. N. Side - band application of systemic granular pesticides for the control of aphids and potato leaf roll virus. Crop Protection, Amsterdam, v. 7, p. 96-105, 1988.

Recebido: 20/08/2007

Received: 08/20/2007

Aprovado: $31 / 10 / 2007$

Approved: 10/31/2007 